\title{
The diagnosis and management of
}

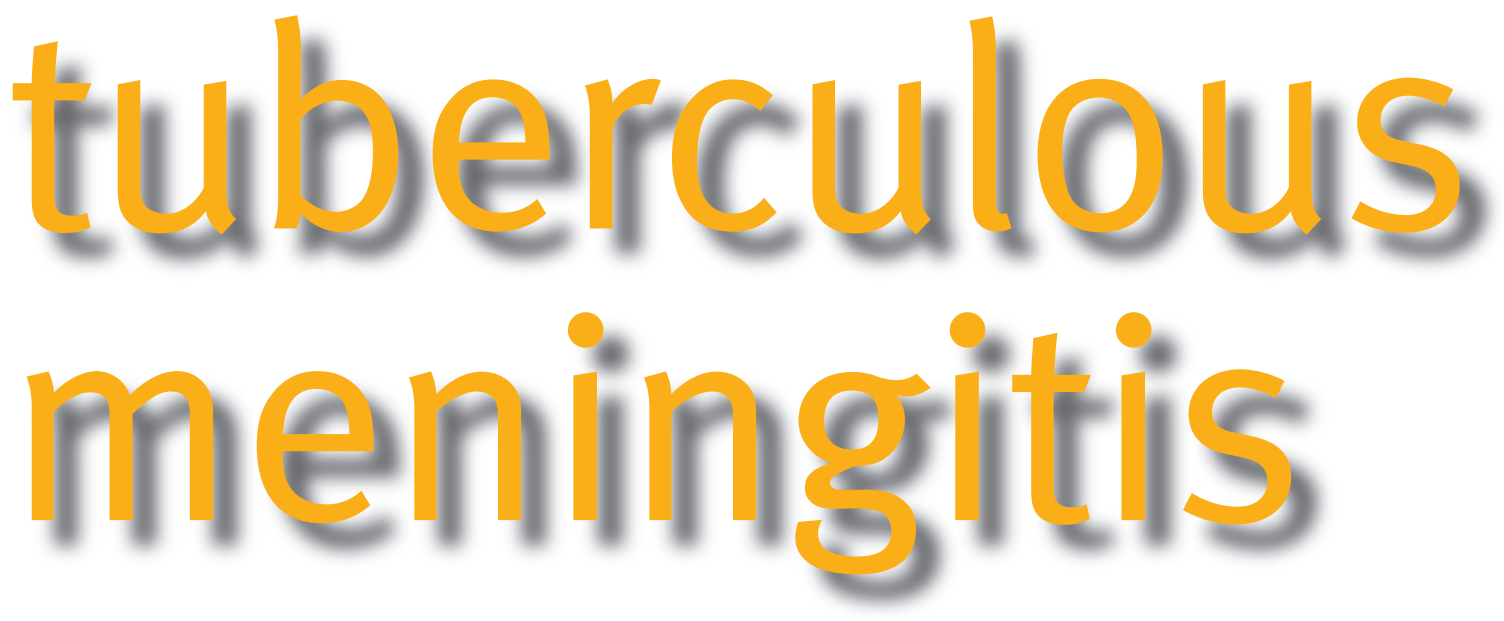

\section{Guy E Thwaites}

Oxford/Wellcome Trust Clinical

Research Unit, 190 Ben Ham

Tu, Ho Chi Minh City, Vietnam;

E-mail: gthwaites@hcm.vnn.vn

Practical Neurology, 2, 250-261

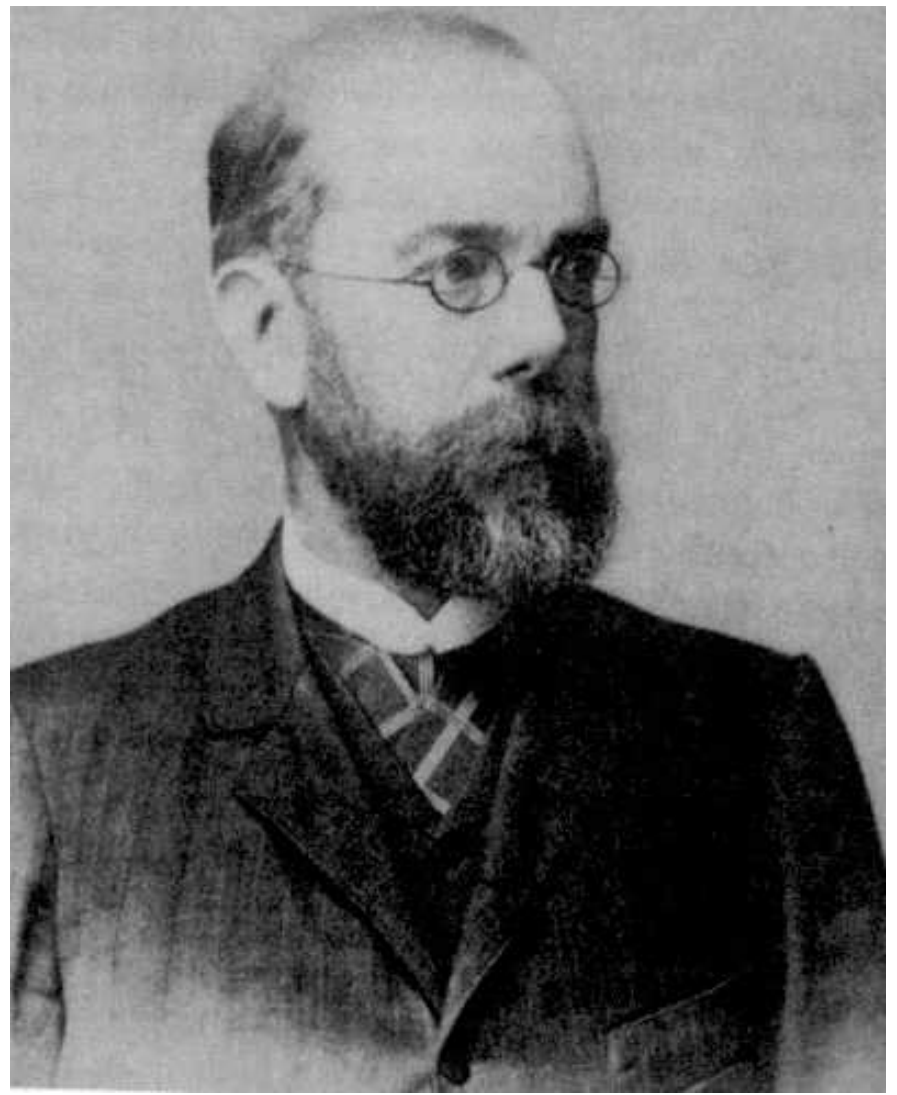

Robert Koch: photograph taken in the 1880 s, around the time of his discovery of the tubercle bacillus (or 'bacille Koch', as it is still called in Vietnam).

\section{INTRODUCTION}

The postmortem observations of Green, published in the Lancet in 1836, were the first to describe the distinct pathological features of tuberculous meningitis and set it apart from the other recognised causes of 'acute hydrocephalus' (Green 1836). The challenge for the physician then lay in distinguishing the disease before death, and delivering the grave prognosis. Thomas Mann captures the full horror of this process in Dr Faustus as the helpless Dr Kurbis presides over the agonizing death of a small child from tuberculous meningitis:

The whole thing lasted scarcely two weeks, including the earliest signs that all was not quite well with the child; from the beginnings no one-I believe no one at all-even dreamed of the horror to come... Kurbis tested the child's eyes, the pupils of which were tiny and showed a tendency to squint. The pulse raced. Muscular contractions developed, and an incipient rigidity of the neck. It was cerebrospinal meningitis, inflammation of the meninges. The good man pronounced the name with a deprecating movement of the head shoulderwards, probably in the hope that they might not know the almost complete powerlessness of medical science in the face of this onslaught.

Tuberculous meningitis was invariably fatal before the advent of anti-tuberculosis chemotherapy. Therefore, the need to diagnose the disorder became a priority after streptomycin was found to reduce mortality of tuberculous meningitis by one third in 1948 (MRC 1948). Progress was rapid over the next 5 years: adding para-aminosalicylic acid to streptomycin reduced mortality to $30 \%$, and the addition of isoniazid to both of these compounds lowered the mortality still more to 
around 20\% (Lorber 1960). Sadly, since the introduction of isoniazid in 1952, the mortality from tuberculous meningitis has altered little. The decline of tuberculosis in the developed world over this period reduced the pressure to discover new drugs and diagnostic methods. As a consequence, in 2002 the optimum drug regimen for treating tuberculous meningitis has not changed since the discovery of rifampicin and pyrazinamide more than 30 years ago, and the best rapid diagnostic method is still the stain developed by Ziehl \& Neelsen in 1883 .

There are three major impediments to reviewing the diagnosis and treatment of tuberculous meningitis. First, much of the literature predates 1966, and therefore cannot be found through Medline. Second, only small numbers of patients have been included in trials. Third, there are few randomised trials anyway. Nevertheless, I will attempt to present the best evidence available to answer two key clinical questions: how to diagnose tuberculous meningitis, and how to treat it.

\section{The diagnosis of tuberculous meningitis}

The diagnosis of tuberculous meningitis is difficult regardless of the resources available to the physician. Because untreated tuberculous meningitis is almost always fatal, it is essential that any diagnostic test is sensitive. The test must also be rapid, because a poor outcome is strongly associated with delayed treatment (Kalita \& Misra 1999). At present, no diagnostic method satisfies both these requirements. The methods available are limited, despite the wealth of possibilities suggested in the literature. Here I will concentrate on clinical diagnosis, and the laboratory methods that are commonly considered world-wide: direct cerebrospinal fluid (CSF) examination for acid-alcohol fast bacilli, CSF culture for Mycobacterium tuberculosis, CSF adenosine deaminase activity, and the detection of mycobacterial nucleic acid in the CSF. The diagnostic place of the tuberculin skin test, chest X-ray, and brain imaging will also be mentioned.

\section{Clinical diagnostic methods}

The clinical features of tuberculous meningitis have been extensively described in a multitude of case reports and clinical series, and are similar to many subacute meningo-encephalitides. Diagnostic uncertainty commonly arises in a comatose patient presenting with a few days of headache, fever and neck-stiffness; undefined

treatment in the community; and a lymphocytic CSF with a low glucose([Fig. 1). The challenge is to decide who requires immediate antituberculosis chemotherapy.

Few studies have attempted to define exactly which clinical features are predictive of the diagnosis of tuberculous meningitis. In one, five presenting clinical features were found to be independently predictive of the diagnosis in 232 children: prodromal stage $\geq 7$ days, optic atrophy, focal neurological deficit, abnormal movements, and CSFleucocytes $<50 \%$ polymorphs (Kumar etal. 1999). The authors developed a simple diagnostic rule: when at least one feature is present diagnostic sensitivity is $98 \%$, specificity 44\%; ifthreeormorefeaturesarepresentsensitivity is reduced to $55 \%$ but specificity rises to $98 \%$. A recent study of 251 Vietnamese adults from our hospital included basic laboratory data from CSF and blood (Thwaites et al. 2002). Five features were predictive of tuberculous meningitis: age, length of history, blood white cell count, total CSF white cell count, and CSF neutrophil percentage. A diagnostic rule developed from these features was $88 \%$ sensitive, and $79 \%$ specific when applied to a further 75 adults (Table 1).

$\begin{array}{ll}\text { VARIABLE } & \text { SCORE } \\ \text { Age (years) } & \\ \quad \geq 36 & +2 \\ \quad<36 & 0 \\ \text { Blood white cell count }\left(10^{3} / \mathrm{mL}\right) & \\ \quad \geq 15000 & +4 \\ \quad<15000 & 0 \\ \text { Duration of illness (days) } & \\ \quad \geq 6 & -5 \\ \quad<6 & 0 \\ \text { CSF total white cell count }\left(10^{3} / \mathrm{mL}\right) & \\ \quad \geq 900 & +3 \\ \quad<900 & 0 \\ \text { CSF percentage neutrophils } & \\ \quad \geq 75 & +4 \\ <75 & 0\end{array}$

Total score $\leq 4$ suggests tuberculous meningitis. Total score $>4$ is against tuberculous meningitis.
Figure 1 A 45-year-old-man was admitted with a 3-day history of coma following 2 weeks of headache and fever. Examination revealed a Glasgow coma score of $8 / 15$, neck stiffness, and a complete right oculomotor nerve palsy. Examination of the CSF showed an opening pressure of $20 \mathrm{~cm} \mathrm{H}_{2} \mathrm{O}, 800$ leucocytes/ $\mathrm{mm}^{3}$ (30\% neutrophils, $70 \%$ lymphocytes), protein $180 \mathrm{mg} /$ $\mathrm{dL}, \mathrm{CSF} /$ blood glucose 0.18 . Gram and Ziehl-Neelsen stains of the CSF were negative. Culture of the CSF on Lowenstein-Jensen media grew Mycobacterium tuberculosis.

Table 1 An admission diagnostic support system for the diagnosis of tuberculous meningitis in adults (Thwaites 2002). Data published by permission of The Lancet. 
These simple diagnostic aids may be of particular benefit to clinicians working with limited microbiological diagnostic facilities, when the majority of adult meningitides are diagnosed and treated on clinical grounds alone. Alternatively, clinicians can use the algorithms to focus the use of specific laboratory diagnostic tests on patients most likely to have tuberculous meningitis.

\section{Laboratory diagnostic methods}

\section{Direct CSF examination for acid-alcohol fast bacilli}

The search for acid-alcohol fast bacilli in clinical specimens has remained the cornerstone of diagnosis ever since Robert Koch first saw the tubercle bacillus in 1882. However, the demonstration of acid-alcohol fast bacilli in the CSF requires diligence and time when excellent results can be achieved, a fact that has long been recognised but often forgotten (Fig. 2). In 1953 Stewart described the method by which her laboratory demonstrated acidalcohol fast bacilli in 91 of 100 consecutive cases of tuberculous meningitis, all of which

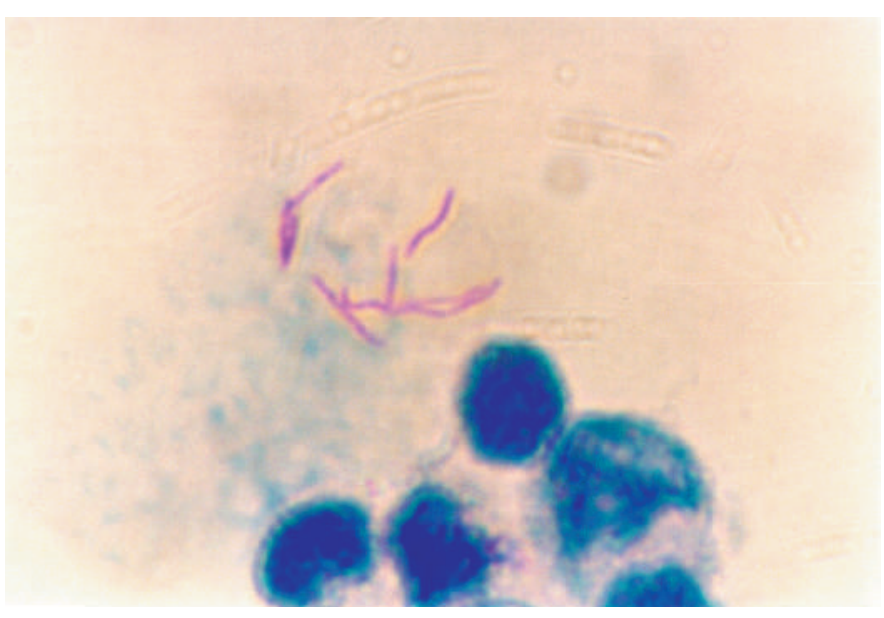

were subsequently confirmed by culture (Stewart 1953). Similar results were reported more recently by Kennedy, who found that bacilli were present in the CSF of 45/52 (87\%) patients with a clinical diagnosis of tuberculous meningitis (Kennedy \& Fallon 1979). Why then do so many laboratories find these results so difficult to reproduce? First, the sensitivity of the direct smear depends on the volume of CSF: $10-20 \mathrm{~mL}$ were examined by Stewart, and as many as four specimens by Kennedy. Clinicians may be reluctant to take the volumes of CSF required to make the diagnosis. Second, the laboratory must undertake a meticulous search for acid-alcohol fast bacilli. Stewart suggested a slide should be examined for between 30 and $120 \mathrm{~min}$, a feat that few modern laboratories would have the staff or patience to complete.

In our hospital we recommend taking 5-10 mL CSF from all patients with suspected tuberculous meningitis. By increasing the volumes of CSF taken, and with careful microscopy, we have increased the diagnostic sensitivity of Ziehl-Neelsen staining from 10\% to $70 \%$.

\section{CSF culture for Mycobacterium tuberculosis}

Although culture of CSF for Mycobacterium tuberculosis is the diagnostic 'gold standard' for tuberculous meningitis, it takes too long for early diagnosis and treatment. The factors that governsensitivityare the same asfor directsmear. Large volumes of CSF yield greater numbers of positive cultures, and diagnostic confirmation by culture can be extremely helpful in patients who have been started on anti-tuberculosis drugs on clinical grounds alone (Fig. 3). In most circumstances the sensitivity and specificity of culture exceeds that of direct smear. However, once treatment has been started the sensitivity of culture falls quickly. Our experience in
Figure 2 Acid alcohol-fast bacilli in the cerebrospinal fluid (purple on Ziehl-Neelsen stain).
Figure 3 Mycobacterium tuberculosis cultured from the CSF on Lowenstein-Jensen media.

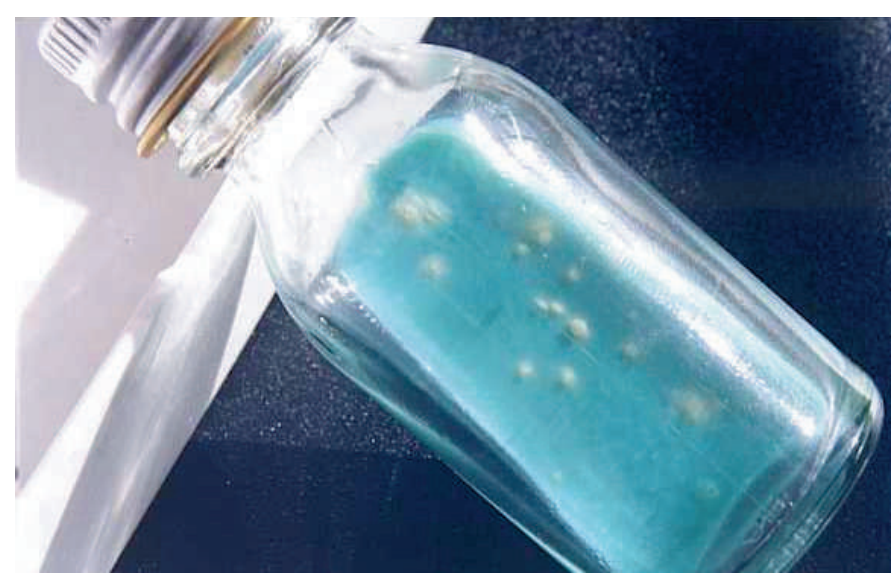


Vietnam suggests that only $5-10 \%$ of patients culture positive before treatment will have positive cultures after $72 \mathrm{~h}$ of four anti-tuberculosis drugs. Although acid-alcohol fast bacilli (presumed to be dead) can be found in the CSF for some days after the start of treatment, they will not grow in culture (Kennedy \& Fallon 1979).

\section{CSF adenosine deaminase activity}

The activity of adenosine deaminase, an enzyme produced by CD4+ lymphocytes and monocytes, is raised in the CSF of patients with tuberculous meningitis. A number of studies have evaluated this as a diagnostic assay (Table 2). The enzyme activity cut-off value used by each study varied from 4 to $10 \mathrm{IU} / \mathrm{mL}$. Diagnostic sensitivity ranges between 44 and $100 \%$, and specificity between 71 and $99 \%$. There are three important problems for clinicians using this assay. First, there is no accepted diagnostic cut-off for the levels of CSF enzyme activity. Second, the assay has not been evaluated in those with HIV infection, which depletes adenosine deaminase producing T-lymphocytes and so may reduce diagnostic sensitivity. Third, patients with lymphomas, malaria, brucellosis, and pyogenic meningitides also have high levels of CSF adenosine deaminase activity. Finally, and in particular, the clinical and laboratory features of partially treated pyogenic meningitis commonly cause diagnostic confusion with tuberculous meningitis - an assay that cannot reliably discriminate between these two conditions is not useful.

\section{The detection of Mycobacterium tuberculosis nucleic acid in the CSF}

The amplification and detection of Mycobacterium tuberculosis nucleic acid from clinical specimens is an attractive diagnostic prospect. Diagnostic specificity is dependent on selecting a region of the genome unique to Mycobacterium tuberculosis, while sensitivity is enhanced by amplification of the selected region from a clinical specimen. This method would appear to be particularly suitable for the diagnosis of tuberculous meningitis when there arefewtuberclebacilliin theCSFandalowchance of contamination with other bacteria. However, despite the development of commercially available assays, the promise is not yet matched by consistent diagnostic performance. Table 3 shows the DNA or RNA target sequence, diagnostic gold standard, and sensitivity and specificity from 20 studies evaluating the use of the polymerase chain reaction (PCR), and other related methods, for the diagnosis of tuberculous meningitis. The variety of targets and 'gold standard' diagnostic criteria is a major impediment to comparing these studies. Few studies have carefully compared the sensitivity of PCR, smear, and culture using large volumes of CSF. Those that have suggest the sensitivity of CSF smear is similar to PCR (Bonington etal. 2000). However, PCR may be more helpful once treatment has begun, when the sensitivity of both smear and culture fall sharply - mycobacterial DNA remains detectable within the CSF for up to one month after the start of treatment (Donald et al. 1993).

The variable specificity of PCR arises for a number of reasons. False-positives may occur through cross-reaction with mycobacteria other than Mycobacterium tuberculosis, from contamination with other clinical samples, and from contaminating DNA in the laboratory. It is this lack of specificity, rather than inadequate sensitivity, that is the main problem using this test (Noordhoek et al. 1996). For this reason, a positive result should be placed within the clinical context before starting treatment, and laboratories must be committed to rigorous internal and external quality control of the use of the assay in different clinical specimens. This is time-consuming and expensive, and is probably only possible within defined diagnostic reference centres. 
Table 3 Studies of the amplification and detection of nucleic acid for the diagnosis of tuberculous meningitis

\begin{tabular}{|c|c|c|c|c|}
\hline STUDY & TARGET & GOLD STANDARD & SENSITIVITY & SPECIFICITY \\
\hline Kaneko et al. (1990) & MBP64 & Clinical & $83 \%$ & $100 \%$ \\
\hline Shankar et al. (1991) & MPB 64 & Clinical & $65 \%$ & $88 \%$ \\
\hline Machado et al. (1994) & $65 \mathrm{KDa}$ antigen & Clinical & $70 \%$ & $100 \%$ \\
\hline Lee et al. (1994) & MPB 64 & Clinical & $83 \%$ & $92 \%$ \\
\hline Folgueira et al. (1994) & IS6110 & Clinical & $100 \%$ & $100 \%$ \\
\hline Liu et al. (1994) & MPB 64 & Clinical & $90 \%$ & $100 \%$ \\
\hline \multirow[t]{3}{*}{ Lee et al. (1994) } & IS6110 & Clinical & $100 \%$ & $38 \%$ \\
\hline & MPB 64 & Clinical & $83 \%$ & $90 \%$ \\
\hline & $65 \mathrm{KDa}$ & Clinical & $83 \%$ & $67 \%$ \\
\hline Kox et al. (1995) & IS6110 & Clinical & $48 \%$ & $100 \%$ \\
\hline Miorner et al. (1995) & IS6110 & Clinical & $54 \%$ & $94 \%$ \\
\hline Scarpellini et al. (1995) & IS6110 & Culture and autopsy & $100 \%$ & $100 \%$ \\
\hline Lin et al. (1995) & MBP64 & Clinical & $70 \%$ & $96 \%$ \\
\hline Seth et al. (1996) & MBP 64 & Clinical & $85 \%$ & $94 \%$ \\
\hline Nguyen et al. (1996) & IS6110 & Clinical & $28 \%$ & $92 \%$ \\
\hline Pfyffer et al. (1996) & rRNA & Clinical & $100 \%$ & $97 \%$ \\
\hline Bonington et al. (1998) & $16 \mathrm{rRNA}^{*}$ & Clinical & $25 \%$ & $100 \%$ \\
\hline Lang et al. (1998) & rRNA & Clinical & $33 \%$ & $100 \%$ \\
\hline \multirow[t]{2}{*}{ Wei et al. (1999) } & IS6110+ & Clinical & $60 \%$ & $67 \%$ \\
\hline & MPB 64 & Clinical & $60 \%$ & $67 \%$ \\
\hline Caws et al. (2000) & IS6110 & Clinical & $39 \%$ & $99 \%$ \\
\hline Bonington et al. (2000) & 16rRNAt & Clinical & $20 \%$ & $100 \%$ \\
\hline \multirow[t]{2}{*}{ Narayanan et al. (2001) } & IS6110 & Clinical & $81 \%$ & $79 \%$ \\
\hline & $\mathrm{TRC}_{4}$ & Clinical & $91 \%$ & $76 \%$ \\
\hline
\end{tabular}

\section{The tuberculin skin test}

The value of the tuberculin skin test for the diagnosis of tuberculous meningitis varies according to age, vaccination with BCG, nutritional status, HIV infection, and the prevalence of tuberculosis. Diagnostic sensitivity is most commonly compromised by the phenomenon of 'anergy' - the failure of those with knowninfection with Mycobacterium tuberculosis to respond to intradermal injection of tuberculin. These false negative results most commonly occur in the elderly, malnourished patients with disseminated tuberculous infection, and those with HIV infection. As these are the very type of individual most at risk of developing tuberculous meningitis, the problems with using the skin test are immediately apparent. Reports suggest only $20 \%$ have a positive tuberculin test (Girgis et al. 1998). However, skin testing may be more useful in children: in one South African series, a reported $86 \%$ had greater than $15 \mathrm{~mm}$ of induration with 5 Units of tuberculin (Donald et al. 1998).
Positive results must also be seen within the context of the tuberculosis prevalence in the area. Individuals with an unrelated illness from high prevalence areas are more likely to have a positive test. The clinician must appreciate these limitations before over-interpreting the results of a skin test. The test is never diagnostic of tuberculous meningitis, but it can be helpful when assessing the likelihood of disease.

\section{The chest $X$-ray and brain imaging}

About half the patients with tuberculous meningitis have a chest X-ray that suggests active or previous pulmonary tuberculosis (Girgis \& Sultan 1998). But, in areas of high tuberculosis prevalence, radiological evidence of previous pulmonary infection is common, and the finding must be interpreted with caution. A small proportion of patients with tuberculous meningitis (5-10\%) have a miliary chest X-ray appearance (Fig. 4).

A number of studies have investigated the role of brain CT - hydrocephalus and contrast enhancing exudates in the basal cisterns are 


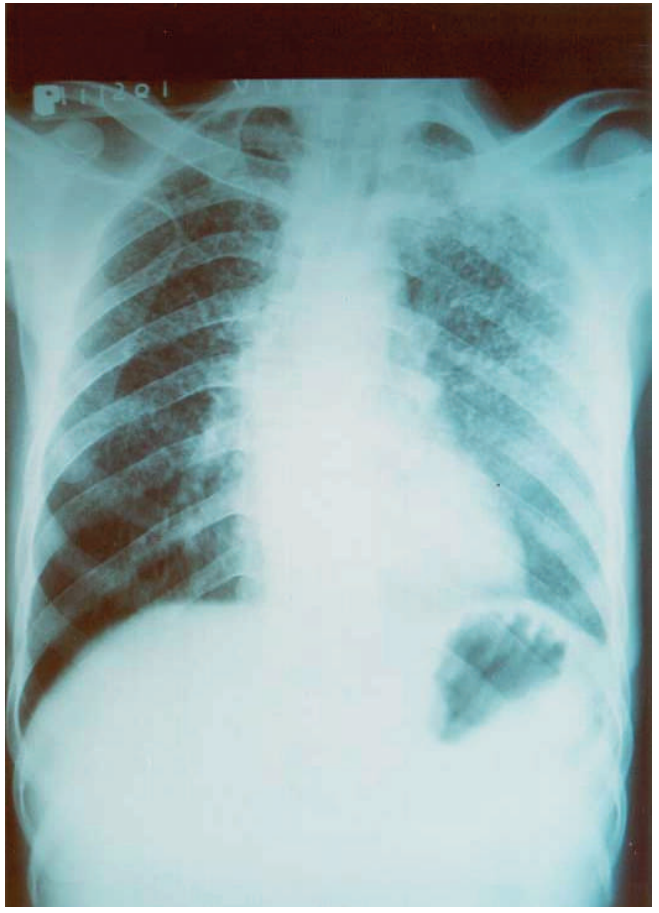

Figure 4 Chest X-ray appearance of miliary

tuberculosis in an adult with tuberculous meningitis.

the most common findings (Fig. 5) (Bhargava et al. 1982; Bullock \& Welchman 1982; Teoh et al. 1989; Hsieh et al. 1992; Kumar et al. 1996; Ozates et al. 2000). However, as autopsy studies have also shown, hydrocephalus is more common in children than adults: brain CT demonstrates severe hydrocephalus in $87 \%$ of children, but only $12 \%$ of adults (Bhargava \& Gupta 1982). Kumar and Kohli compared the CT appearances of 94 children with tuberculous meningitis and 52 with pyogenic meningitis. Basal meningeal enhancement or tuberculoma, or both, was $89 \%$ sensitive and $100 \%$ specific for the diagnosis of tuberculous meningitis (Kumar \& Kohli 1996). Teoh and Humphries even suggested 'a normal scan in a drowsy patient excludes the diagnosis of tuberculous meningitis' (Teoh \& Humphries 1989). However, this is probably untrue, particularly in adults, because in a recent series the scan was normal in 35 of 289 (12\%) patients with tuberculous meningitis and not all were fully conscious. The abnormalities in this series were hydrocephalus ( $80 \%$ of children, $43 \%$ of adults), parenchymal enhancement (26\% of children, $8 \%$ of adults), contrast enhancement of basal cisterns ( $15 \%$ of children, $23 \%$ of adults), cerebral infarct and focal or diffuse brain oedema ( $14 \%$ of children, $13 \%$ of adults),

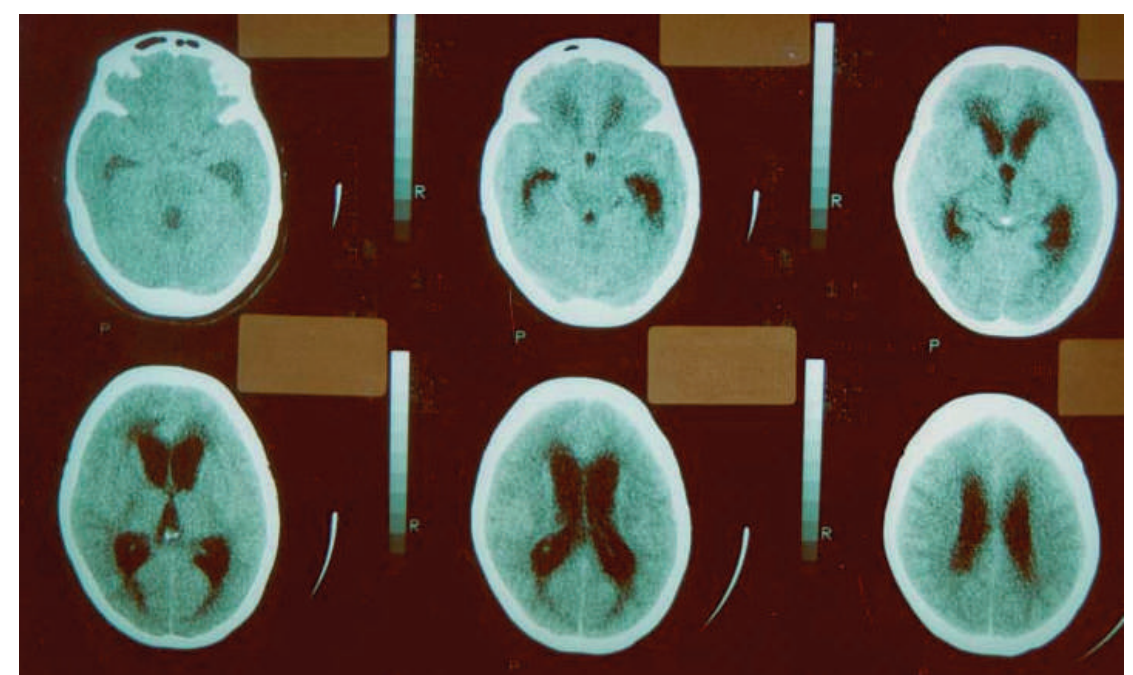

(a)

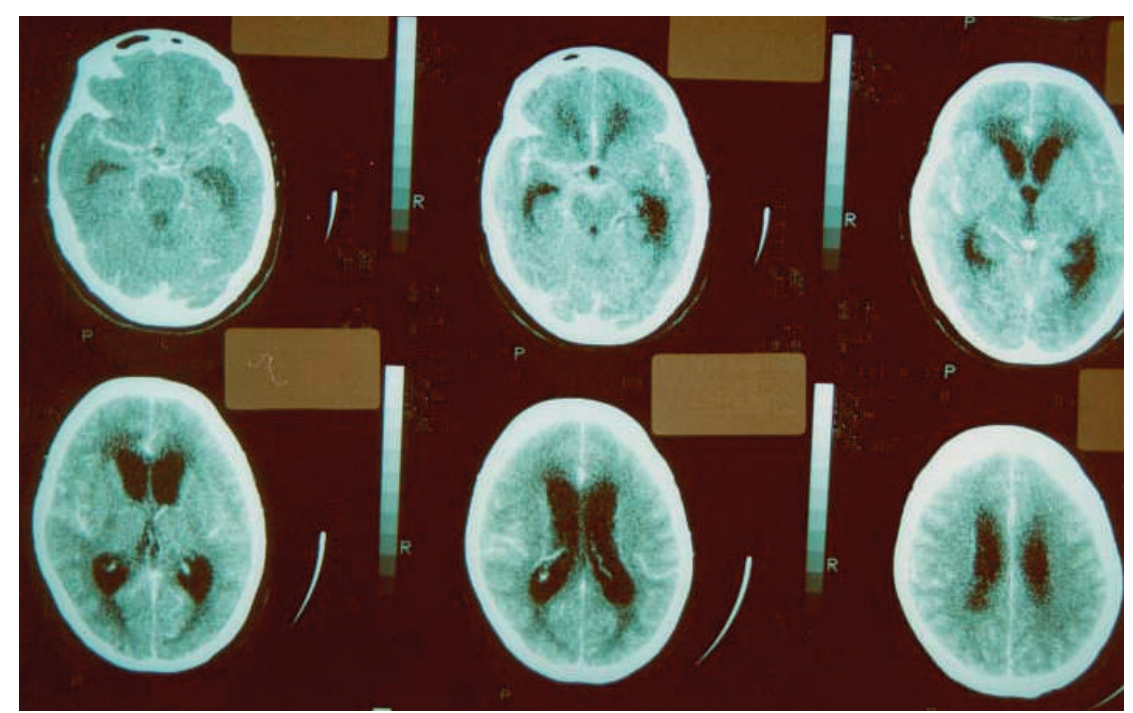

(b)

Figure 5 Brain $\mathrm{CT}$ of an adult with culture confirmed tuberculous meningitis. (a) precontrast scan showing dilated ventricles. (b) postcontrast scan demonstrating enhancement of the basal cisterns.

and tuberculoma ( $4 \%$ of children, $7 \%$ of adults) (Ozates \& Kemaloglu 2000).

Magnetic resonance imaging (MRI) may provide more diagnostic information than CT when assessing space-occupying lesions, infarcts, and the extent of the inflammatory exudate (Tartaglione et al. 1998). However, data on the diagnostic sensitivity and specificity of MRI for tuberculous meningitis are limited - those studies that do exist are small, and only include a highly selected patient population. However, MRI is probably more sensitive than CT for detecting the diverse intracranial pathology of tuberculous meningitis. But neither investigation will reveal appearances specific for tuberculous meningitis 
- cryptococcal meningitis, cytomegalovirus encephalitis, sarcoidosis, meningeal metastases, and lymphoma may all produce similar findings on brain imaging.

\section{THE MANAGEMENT OF TUBERCULOUS MENINGITIS}

The outcome of tuberculous meningitis remains poor, despitetheavailabilityofhighly effective chemotherapyagainst pulmonarytuberculosis. Many recent series report mortality greater than 20\%, and severe neurological sequelae in approximately $30 \%$ of survivors (Hosoglu et al. 2002; Girgis \& Sultan 1998). The factors influencing prognosis have been defined by a number of studies. All attest to the importance of early treatment before the onset of coma. A recent retrospective study of 434 Turkish adults revealed convulsions, coma, anddelayed or interrupted treatment to be independent predictors of mortality. Extra-meningeal tuberculosis, cranial nerve palsy, focal weakness, multiple neurological abnormalities, and drowsiness independently predicted later neurological disability (Hosoglu 2002). The message is simple: do not delay treatment in those in whom the diagnosis is suspected, although the limitations of current diagnostic methods will result in many patients starting treatment without a confirmed diagnosis.

\section{Anti-tuberculosis chemotherapy}

\section{The drugs}

The treatment of tuberculous meningitis followsthemodelofshortcoursechemotherapy for pulmonary tuberculosis - an intensive phase of treatment, followed by a continuation phase. But, unlike pulmonary tuberculosis, the optimal drug regimen and duration of each phase are uncertain. The British Thoracic Society (BTS 1998), the Infectious Diseases Society of America, and the American
Thoracic Society (ATS 1994) recommend that all patients start on isoniazid, rifampicin, and pyrazinamide, although neither rifampicin nor pyrazinamide have been shown to improve outcome or reduce duration of treatment for tuberculous meningitis. Isoniazid is believed to be critical because it penetrates the CSF freely and has potent early bactericidal activity.

Choosing the fourth drug of the intensive phase is more difficult. The BTS recommend either streptomycin or ethambutol, although neither penetrates the CSF well in the absence of inflammation, and both can produce significant adverse reactions (see below). Streptomycin should not be given to those who are pregnant or have renal impairment. Intra-thecal streptomycin is no longer used, although this route of administration is being revisited for the treatment of multidrug resistant cases. Some centres, notably in South Africa, advocate ethionamide, which penetrates healthy and inflamed meninges (Donald \& Seifart 1989). However, despite theoretical attractions, there are no data demonstrating any clear advantage of one drug over another. The prevalence and patterns of drug resistance may influence the choice of drug. For example, streptomycin resistance is common in Vietnam, particularly in the HIV population, and ethambutol is favoured as a consequence.

Isoniazid and rifampicin should be given for the continuation phase of treatment. Although there are no data to suggest pyrazinamide improves outcome, some authorities suggest it should accompany these drugs given the high CSF concentrations achieved throughout the course of the disease (Humphries 1992).

Tuberculous meningitis caused by organisms resistant to both isoniazid and rifampicin (multidrug resistance) presents a formidable therapeutic challenge. For suspected, or proven, multidrug resistant pulmonary tuberculosis, the World Health Organization recommend an aminoglycoside (kanamycin, amikacin, or 
capreomycin), ethionamide, pyrazinamide, and ofloxacin for the initial phase of treatment (Crofton et al. 1997). There are no equivalent recommendations for multidrug resistant tuberculous meningitis, and few data are available on the CSF penetration and effectiveness of possible agents. Ethionamide, prothionamide and cycloserine have been reported to penetrate the blood-brain barrier and may be effective. The combination of intrathecal amikacin and levofloxacin has also been suggested (Berning et al. 2001). Until more data become available, the treatment of patients with multidrug resistant tuberculous meningitis should be guided by drug resistance profiles in individual patients, and the predicted CSF penetration of candidate drugs.

\section{The dose}

The BTS recommendations for the dosages of the standard anti-tuberculosis drugs are shown in Table 4. Some authors have suggested using doses of isoniazid greater than $5 \mathrm{mg} / \mathrm{kg}$ for the treatment of tuberculous meningitis (Humphries 1992).Thepotent earlybactericidal effect of isoniazid, and the uncertain CSF penetration of other drugs in the standard regimen, makes this an attractive suggestion. However, at standard doses isoniazid achieves CSF levels 10-15 times the minimum inhibitory concentration of Mycobacterium tuberculosis (Kaojarern \& Supmonchai 1991), and there are no data to suggest higher doses improve outcome or shorten treatment in adults with tuberculous meningitis.

\section{Duration}

Tuberculous meningitis should be treated for at least 6 months. However, it is unclear whether more prolonged treatment is required. The BTS recommend 12 months in uncomplicated cases, extending to 18 months should pyrazinamide

Table 4 The recommended daily dosages of standard anti-tuberculosis drugs (BTS 1998)

\begin{tabular}{|c|c|c|}
\hline DRUG & CHILDREN & ADULTS \\
\hline Isoniazid & $5 \mathrm{mg} / \mathrm{kg}$ & $300 \mathrm{mg}$ \\
\hline Rifampicin & $10 \mathrm{mg} / \mathrm{kg}$ & $\begin{array}{l}450 \mathrm{mg} \text { if weight }<50 \mathrm{~kg} \\
600 \mathrm{mg} \text { if weight }>50 \mathrm{~kg}\end{array}$ \\
\hline Pyrazinamide & $35 \mathrm{mg} / \mathrm{kg}$ & $\begin{array}{l}1.5 \mathrm{~g} \text { if weight }<50 \mathrm{~kg} \\
2.0 \mathrm{~g} \text { if weight }>50 \mathrm{~kg}\end{array}$ \\
\hline Ethambutol & $15 \mathrm{mg} / \mathrm{kg}$ & $15 \mathrm{mg} / \mathrm{kg}$ \\
\hline Streptomycin & $15 \mathrm{mg} / \mathrm{kg}$ & $15 \mathrm{mg} / \mathrm{kg}(\max 1 \mathrm{~g})$ \\
\hline
\end{tabular}

be omitted (BTS 1998). Treatment for 12 months is probably an over-estimation of the time required for bacterial cure, and there is some evidence to suggest shorter courses are effective. A recent systematic review concluded that 6 months of anti-tuberculosis drugs for tuberculous meningitis is probably sufficient, provided the likelihood of drug resistance is low (van Loenhout-Rooyackers et al. 2001). Disease severity, drug toxicity and patient compliance should all be considered when deciding the duration of treatment. Although 6 months of treatment is probably effective for most patients, in Vietnam we use four drugs for 3 months, followed by three drugs for another 6 months. Treatment is extended in those unable to tolerate the first-line drugs, and those with persistent symptoms, signs and inflammatory CSF changes after 9 months of treatment.

\section{Adjunctive corticosteroids}

Corticosteroids for the treatment of tuberculous meningitis are still controversial. A recent Cochrane review included six randomised trials involving a total of 595 patients (Prasad et al.2000). Those receiving corticosteroids had a lower death rate (relative risk $0.79,95 \%$ CI 0.65 to 0.97 ) and a reduced risk of death or severe neurological sequaelae (relative risk $0.58,95 \%$ CI 0.38 to 0.88 ). Subgroup analysis suggested a beneficial effect on mortality in children, but inconclusive results in adults partly due to the small numbers of adult patients. The reviewers concluded that adjunctive corticosteroids may be of benefit in patients with tuberculous meningitis, but the evidence is limited. The existing studies are small with poor allocation concealment, and publication bias may account for the results favouring corticosteroids.

There are no conclusive data regarding the choice of corticosteroid, dose or duration of treatment. A reduction in mortality has been suggested using either prednisolone or dexamethasone for at least the first 3 weeks of antituberculosis chemotherapy, followed by a similar period, as the dose is decreased to zero (Girgis et al. 1991; Schoeman et al. 1997). These trials gave children $4 \mathrm{mg} / \mathrm{kg} /$ day of prednisolone, or $12 \mathrm{mg} /$ day of dexamethasone, and 
adults $16 \mathrm{mg} /$ day of dexamethasone. To help resolve this issue we are doing a further trial in Vietnam.

\section{Response to treatment}

Ninety percent of deaths from tuberculous meningitis occur in the first month of treatment (Girgis \& Sultan 1998). The response to treatment is usually slow, and the patients may fluctuate. Indeed, a rapid and sustained response over a few days suggests the diagnosis is wrong. Headache often persists for many weeks, even in uncomplicated cases. Fever rarely disappears within a week, and frequently lingers for 6-8 weeks. The degree of neck rigidity at presentation varies considerably, but can take 4-6 weeks to resolve. The CSF mirrors the slow clinical response - cell counts are raised for 1-2 months, glucose remains low for a similar period, and total CSF protein can rise before falling slowly over many months (Schoeman et al.2001).

Transient episodes of high fever, worsening headache, and increased neck rigidity can occur during the first two months of treatment, particularly in those with more severe disease. Distinguishing self-limiting events from the onset of more serious complications is difficult. In our experience, new focal neurological signs, or a fall in conscious level, rarely accompany these transient deteriorations. Brain imaging should be arranged urgently if new clinical signs develop during treatment. Hydrocephalus, cerebral infarction, the expansion of intracranial tuberculoma, and poor adherence to therapy, are the foremost reasons for severe acute deterioration.

\section{Relapse, paradoxical treatment reactions, and adverse drug events}

There are many reasons for clinical deterioration after the onset of appropriate chemotherapy for tuberculous meningitis (Table 5). Paradoxical treatment reactions are a well-recognised phenomenon in all forms of tuberculosis. Although the pathogenesis remains unclear, such reactions are characterised by an intense inflammatory response. Data on the frequency and timing of these events in tuberculous meningitis are restricted to occasional case reports. The expansion of intracranial tuberculoma after the start of anti-tuberculosis drugs is the most widely reported example, and can occur at any time during treatment (Afghani \& Leiberman 1994). Most authors suggest treatment with prolonged high-dose corticosteroids, although there are no controlled trials to support this recommendation.

Adverse reactions to anti-tuberculosis drugs are a common problem, and can have a devastating effect on the outcome. Table 6 presents the common, the rare, and the neurological adverse reactions to the main anti-tuberculosis drugs. Hepatic toxicity is the most important. The BTS recommend stopping isoniazid, rifampicin and pyrazinamide immediatelyifthetransaminasesrisetofivetimes normal, or the bilirubin level rises (BTS 1998). In most forms of tuberculosis a short period without treatment does not affect outcome. Unfortunately, treatment interruptions of this type in tuberculous meningitis are an independent predictor of death as they often lead to relapse with neurological deterioration (Hosoglu 2002). Streptomycin and ethambutol should both be given in these circumstances, and isoniazid and rifampicin restarted as soon as possible. Treatment should be extended if the patient cannot adhere to the conventional ninemonth regimen

\section{Neurosurgery}

Neurosurgical intervention may be indicated for the following complications of tuberculous meningitis: hydrocephalus, tuberculoma and abscess formation. Unfortunately, there are no published randomised trials of surgery for any of these complications. Hydrocephalus occurs more commonly in children than adults, but

Table 5 Common reasons for clinical deterioration in patients with tuberculous meningitis

$\begin{array}{ll}\text { EARLY DETERIORATION (1-3 MONTHS) } & \text { LATE DETERIORATION (3-12 MONTHS) } \\ \text { Prescription error } & \text { Prescription error } \\ \text { Poor patient compliance } & \text { Poor patient compliance } \\ \text { Drug reaction requiring either reduction } & \text { Paradoxical treatment reactions } \\ \text { in dose, or withdrawal of all or some } & \\ \quad \text { of the drugs (see Table 6) } & \\ \text { Paradoxical treatment reactions } & \end{array}$


Table 6 Adverse reactions to anti-tuberculosis drugs (BTS 1998; Holdiness 1987)

\begin{tabular}{|c|c|c|c|}
\hline & COMMON & RARE & NEUROLOGICAL \\
\hline \multirow[t]{7}{*}{ Isoniazid } & Hepatitis & Haemolytic anaemia & Peripheral neuropathy \\
\hline & & Aplastic anaemia & Convulsions \\
\hline & & Sideroblastic anaemia & Optic neuritis \\
\hline & & Agranulocytosis & Mania/psychosis \\
\hline & & Lupoid reactions & \\
\hline & & Arthralgia & \\
\hline & & Gynaecomastia & \\
\hline \multirow[t]{3}{*}{ Rifampicin } & Hepatitis & Haemolytic anaemia & Headaches \\
\hline & Thrombocytopenia & Acute renal failure & Confusion \\
\hline & Fever & & Drowsiness \\
\hline \multirow[t]{5}{*}{ Pyrazinamide } & Hepatitis & Gout & \\
\hline & Anorexia & Photosensitivity & \\
\hline & Flushing & & \\
\hline & Arthralgia & & \\
\hline & Hyperuricaemia & & \\
\hline \multirow[t]{3}{*}{ Ethambutol } & Arthralgia & Hepatitis & Retrobulbar neuritis \\
\hline & & Rash & Peripheral neuropathy \\
\hline & & & Confusion \\
\hline \multirow[t]{3}{*}{ Streptomycin } & Vertigo & & Neuromuscular block \\
\hline & Deafness & & \\
\hline & Acute renal failure & & \\
\hline
\end{tabular}

is the commonest reason for neurosurgical referral in both groups. A recent series reported that ventriculo-peritoneal shunting was performed in 30\% (65/217) of children with hydrocephalus complicating tuberculous meningitis (Lamprecht et al. 2001). After 6 months of treatment, $12 \%$ had died, and $45 \%$ had severe sequelae. It is difficult to predict which patients with hydrocephalus will benefit from shunts. Clinical response to external ventricular drainage has been assessed for this purpose, but failed to predict benefit, so perhaps early ventriculo-peritoneal shunting should be considered in all patients with hydrocephalus (Palur et al. 1991; Mathew et al. 1998). But this aggressive approach does not acknowledge the significant complications of shunt surgery, the variable resources and experience of surgical units, and the lack of trials demonstrating any benefit.

\section{CONCLUSIONS}

- The early diagnosis and treatment of tuberculous meningitis saves lives.

- Microscopy for acid-alcohol fast bacilli is still the best rapid diagnostic test, although the sensitivity depends on the volume of CSF examined.

- Treatment with four drugs should begin as soon as possible.

- Adjunctive corticosteroids should be considered for patients presenting in coma.

- Brain imaging should be performed if the patient deteriorates, and the complications of hydrocephalus, infarction, and tuberculoma considered. A neurosurgical opinion should be sought in those with hydrocephalus who are failing medical therapy.

\section{REFERENCES}

Afghani B \& Leiberman JM (1994) Paradoxical enlargement or development of intracranial tuberculomas during therapy: case report and review. Clinical Infectious Diseases, 19, 1092-9.

American Thoracic Society (1994) Treatment of tuberculosis and tuberculosis infection in adults and children. American Thoracic Society. American Journal of Respiratory Critical Care Medicine, 149, 1359-74.

Baro M, Acevedo L \& Lagos ME (1996) [Usefulness of adenosine deaminase determination in cerebrospinal 
fluid for the diagnosis of meningeal tuberculosis: 4 years experience at a public hospital]. Review Medical Chili, 124, 319-26.

Berning SE, Cherry TA \& Iseman MD (2001) Novel treatment of meningitis caused by multidrugresistant Mycobacterium tuberculosis with intrathecal levofloxacin and amikacin: case report. Clinical Infectious Diseases, 32, 643-6.

Bhargava S, Gupta AK \& Tandon PN (1982) Tuberculous meningitis - a CT study. British Journal of Radiology, 55, 189-96.

Bonington A, Strang JI \& Klapper PE et al. (1998) Use of Roche AMPLICOR Mycobacterium tuberculosis PCR in early diagnosis of tuberculous meningitis. Journal of Clinical Microbiology, 36, 1251-4.

Bonington A, Strang JI, Klapper PE et al. (2000) TB PCR in the early diagnosis of tuberculous meningitis: evaluation of the Roche semi-automated COBAS Amplicor MTB test with reference to the manual Amplicor MTB PCR test. Tubercule and Lung Disease, 80, 191-6.

British Thoracic Society (1998) Chemotherapy and management of tuberculosis in the United Kingdom: recommendations 1998:Joint TuberculosisCommittee of the British Thoracic Society. Thorax, 53, 536-48.

Bullock MR \& Welchman JM (1982) Diagnostic and prognostic features of tuberculous meningitis on CT scanning. Journal of Neurology, Neurosurgery and Psychiatry, 45, 1098-101.

Caws M, Wilson SM, Clough C et al. (2000) Role of IS6110-targeted PCR, culture, biochemical, clinical, and immunological criteria for diagnosis of tuberculous meningitis. Journal of Clinical Microbiology, 38, 3150-5.

Coovadia YM, Dawood A, Ellis ME et al. (1986) Evaluation of adenosine deaminase activity and antibody to Mycobacterium tuberculosis antigen 5 in cerebrospinal fluid and the radioactive bromide partition test for the early diagnosis of tuberculosis meningitis. Archives of the diseases of childhood, 61, $428-35$.

Crofton J, Chaulet P \& Maher D (1997) Guidelines for the Management of Drug-Resistant Tuberculosis. Geneva, WHO TB.

Donald PR, Schoeman JF, Van Zyl LE et al. (1998) Intensive short course chemotherapy in the management of tuberculous meningitis. International Journal of Tuberculosis and Lung Disease, 2, 704-11.

Donald PR \& Seifart HI (1989) Cerebrospinal fluid concentrations of ethionamide in children with tuberculous meningitis. Journal of Pediatrics, 115, 483-6.

Donald PR, Victor TC, Jordaan AM et al. (1993) Polymerase chain reaction in the diagnosis of tuberculous meningitis. Scandinavian Journal of Infectious Diseases, 25, 613-7.

Folgueira L, Delgado R, Palenque E et al. (1994) Polymerase chain reaction for rapid diagnosis of tuberculous meningitis in AIDS patients. Neurology, 44, 1336-8.

Gambhir IS, Mehta M, Singh DS et al. (1999) Evaluation of CSF-adenosine deaminase activity in tubercular meningitis. Journal of Association Physicians India, $47,192-4$.
Girgis NI, Farid Z, Kilpatrick ME et al. (1991) Dexamethasone adjunctive treatment for tuberculous meningitis. Pediatric Infectious Diseases Journal, 10, 179-83.

Girgis NI, Sultan Y, Farid Z et al. (1998) Tuberculosis meningitis, Abbassia Fever Hospital - Naval Medical Research Unit, 3 - Cairo, Egypt, from 1976 to 1996. American Journal of Tropical Medicine and Hygiene, $\mathbf{5 8}, 28-34$.

Green PH (1836) Tubercular meningitis. Lancet, ii, $232-5$.

Holdiness MR (1987) Neurological manifestations and toxicities of the antituberculosis drugs. A Review. Medical Toxicology, 2, 33-51.

Hosoglu S, Geyik MF, Balik I et al. (2002) Predictors of outcome in patients with tuberculous meningitis. International Journal of Tuberculosis and Lung Disease, 6, 64-70.

Hsieh FY, Chia LG \& Shen WC (1992) Locations of cerebral infarctions in tuberculous meningitis. Neuroradiology, 34, 197-9.

Humphries M (1992) The management of tuberculous meningitis. Thorax, 47, 577-81.

Kalita J \& Misra UK. (1999) Outcome of tuberculous meningitis at 6 and 12 months: a multiple regression analysis. International Journal of Tuberculosis and Lung Disease, 3, 261-5.

Kaneko K, Onodera O, Miyatake T et al. (1990) Rapid diagnosis of tuberculous meningitis by polymerase chain reaction (PCR). Neurology, 40, 1617-8.

Kaojarern S, Supmonchai K, Phuapradit P et al. (1991) Effect of steroids on cerebrospinal fluid penetration of antituberculous drugs in tuberculous meningitis. Clinical Pharmacology and Therapeutics, 49, 6-12.

KennedyDH \& Fallon RJ (1979) Tuberculous meningitis. Journal of the American Medical Association, 241, 264-8.

Kox LF, Kuijper S \& Kolk AH (1995) Early diagnosis of tuberculous meningitis by polymerase chain reaction. Neurology, 45, 2228-32.

Kumar R, Kohli N, Thavnani H et al. (1996) Value of CT scan in the diagnosis of meningitis. Indian Pediatrics, 33, 465-8.

Kumar R, Singh SN, Kohli N (1999) A diagnostic rule for tuberculous meningitis. Archives of the diseases of childhood, 81, 221-4.

Lamprecht D, Schoeman J, Donald P et al. (2001) Ventriculoperitoneal shunting in childhood tuberculous meningitis. British Journal of Neurosurgery, 15, 119-25.

Lang AM, Feris-Iglesias J, Pena C et al. (1998) Clinical evaluation of the General-Probe Amplified Direct Test for detection of Mycobacterium tuberculosis complex organisms in cerebrospinal fluid. Journal of Clinical Microbiology, 36, 2191-4.

Lee BW, Tan JA, Wong SC et al. (1994) DNA amplification by the polymerase chain reaction for the rapid diagnosis of tuberculous meningitis. Comparison of protocols involving three mycobacterial DNA sequences, IS6110, $65 \mathrm{kDa}$ antigen, and MPB64. Journal of Neurological Science, 123, 173-9.

Lee BW, Tan JA, Wong SC et al. (1994) The diagnosis of tuberculous meningitis using the polymerase chain reaction. Singapore Medical Journal, 35, 360-3. 
Lin JJ, Harn HJ, Hsu YD et al. (1995) Rapid diagnosis of tuberculous meningitis by polymerase chain reaction assay of cerebrospinal fluid. Journal of Neurology, 242, 147-52.

Liu PY, Shi ZY, Lau YJ et al. (1994) Rapid diagnosis of tuberculous meningitis by a simplified nested amplification protocol. Neurology, 44, 1161-4.

van Loenhout-Rooyackers JH, Keyser A, Laheij RJ et al. (2001) Tuberculous meningitis: is a 6-month treatment regimen sufficient? International Journal of Tuberculosis and Lung Disease, 5, 1028-35.

Lopez-Cortes LF, Cruz-Ruiz M, Gomez-Mateos J et al. (1995) Adenosine deaminase activity in the CSF of patientswithasepticmeningitis:utilityin the diagnosis of tuberculous meningitis or neurobrucellosis. Clinical Infectious Diseases, 20, 525-30.

LorberJ (1960) The treatment of tuberculous meningitis. British Medical Journal, i, 1309-12.

Machado LR, Livramento JA, Bydlowski SP et al. (1994) Polymerase chain reaction in the diagnosis of tuberculous meningitis. Preliminary report. Arq Neuropsiquiatrics, 52, 445-6.

Mann T (1947) Dr Faustus. London: David Campbell;

Mann MD, Macfarlane CM, Verburg CJ et al. (1982) The bromide partition test and CSF adenosine deaminase activity in the diagnosis of tuberculosis meningitis in children. South African Medical Journal, 62, 431-3.

Mathew JM, Rajshekhar V, Chandy MJ (1998) Shunt surgery in poor grade patients with tuberculous meningitis and hydrocephalus. effects of response to external ventricular drainage and other variables on long term outcome. Journal of Neurology, Neurosurgery and Psychiatry, 65, 115-8.

Miorner H, Sjobring U, Nayak P et al. (1995) Diagnosis of tuberculous meningitis: a comparative analysis of 3 immunoassays, an immune complex assay and the polymerase chain reaction. Tubercule and Lung Disease, 76, 381-6.

Mishra OP, Loiwal V, Ali Z et al. (1995) Cerebrospinal fluid adenosine deaminase activity and C-reactive protein in tuberculous and partially treated bacterial meningitis. Indian Pediatrics, 32, 886-9.

Mishra OP, Loiwal V, Ali Z et al. (1996) Cerebrospinal fluid adenosine deaminase activity for the diagnosis of tuberculous meningitis in children. Journal of Tropical Pediatrics, 42, 129-32.

Medical Research Council (1948) Streptomycin treatment of tuberculous meningitis. British Medical Journal, i, 582-97.

Narayanan S, Parandaman V, Narayanan PR et al. (2001) Evaluation of PCR using TRC (4) and IS6110 primers in detection of tuberculous meningitis. Journal of Clinical Microbiology, 39, 2006-8.

Nguyen LN, Kox LF, Pham LD et al. (1996) The potential contribution of the polymerase chain reaction to the diagnosis of tuberculous meningitis. Archives of Neurology, 53, 771-6.

Noordhoek GT, van Embden JD, Kolk AH (1996) Reliability of nucleic acid amplification for detection of Mycobacterium tuberculosis: an international collaborative quality control study among 30 laboratories. Journal of Clinical Microbiology, 34, 2522-5.
Ozates M, Kemaloglu S, Gurkan F et al. (2000) CT of the brain in tuberculous meningitis. A review of 289 patients. Acta Radiologica, 41, 13-7.

Palur R, Rajshekhar V, Chandy MJ et al. (1991) Shunt surgery for hydrocephalus in tuberculous meningitis: a long-term follow-up study. Journal of Neurosurgery, 74, 64-9.

Pfyffer GE, Kissling P, Jahn EM et al. (1996) Diagnostic performance of amplified Mycobacterium tuberculosis direct test with cerebrospinal fluid, other nonrespiratory, and respiratory specimens. Journal of Clinical Microbiology, 34, 834-41.

Prasad K, Volmink J, Menon GR (2000) Steroids for treating tuberculous meningitis. Cochrane Database, Syst Rev: 3.

Ribera E, Martinez-Vazquez JM, Ocana I et al. (1987) Activity of adenosine deaminase in cerebrospinal fluid for the diagnosis and follow-up of tuberculous meningitis in adults. Journal of Infectious Diseases, 155, 603-7.

Rohani MY, Cheong YM \& Rani JM (1995) The use of adenosine deaminase activity as a biochemical marker for the diagnosis of tuberculous meningitis. Malaysian Journal of Pathology, 17, 67-71.

Scarpellini P, Racca S, Cinque P et al. (1995) Nested polymerase chain reaction for diagnosis and monitoring treatment response in AIDS patients with tuberculous meningitis. Aids, 9, 895-900.

Schoeman JF, Elshof JW, Laubscher JA et al. (2001) The effect of adjuvant steroid treatment on serial cerebrospinal fluid changes in tuberculous meningitis. Annals of Tropical Paediatrics, 21, 299-305.

Schoeman JF, Van ZylLE, Laubscher JAet al. (1997) Effect of corticosteroids on intracranial pressure, computed tomographic findings, and clinical outcome in young children with tuberculous meningitis. Pediatrics, 99, 226-31.

Seth P, Ahuja GK, Bhanu NV et al. (1996) Evaluation of polymerase chain reaction for rapid diagnosis of clinically suspected tuberculous meningitis. Tubercule and Lung Disease, 77, 353-7.

Shankar P, Manjunath N, Mohan KK et al. (1991) Rapid diagnosis of tuberculous meningitis by polymerase chain reaction. Lancet, 337, 5-7.

Stewart SM (1953) The bacteriological diagnosis of tuberculous meningitis. Journal of Clinical Pathology, 6, 241-2.

Tartaglione T, Di Lella GM, Cerase A et al. (1998) Diagnostic imaging of neurotuberculosis. Rays, 23, 164-80.

Teoh R, Humphries MJ, Hoare RD et al. (1989) Clinical correlation of CT changes in 64 Chinese patients with tuberculous meningitis. Journal of Neurology, 236, $48-51$.

Thwaites GE, Chau TTH, Strepniewska K et al. (2002) Diagnosis of adult tuberculous meningitis by use of clinical and laboratory features. The Lancet, in press.

Wei CY, Hwang JJ, Chu CH et al. (1999) Detection and identification of Mycobacterium tuberculosis by nested PCR assays in cerebrospinal fluid samples from patients with suspected tuberculous meningitis. Kaohsiung Journal of Medical Science, 15, 475-83. 